\title{
An atypical cause of right lower abdominal pain: amoebiasis, a family cluster
}

\author{
T. De Somer ${ }^{1}$, D. Baert ${ }^{1}$, M. Deceuninck ${ }^{2}$, C. Van Steenkiste ${ }^{1,3}$, D. Marichal ${ }^{4}$, C. Gabriel ${ }^{5}$, E. Vanderstraeten ${ }^{1}$, P. Dewint $^{1,3}$
}

(1) Department of Gastroenterology and Hepatology, Maria Middelares Hospital, Buitenring Sint-Denijs 30, 9000 Gent, Belgium ; (2) Department of Gastroenterology and Hepatology, Sint-Vincentius Hospital, Schutterijstraat 34, 9800 Deinze, Belgium ; (3) Department of Gastroenterology and Hepatology, University Hospital Antwerp, Wilrijkstraat 10, 2650 Edegem, Belgium ; (4) Department of Gastroenterology and Hepatology, Sint-Lucas Hospital Gent, Groenebriel 1, 9000 Gent, Belgium ; (5) Department of Histopathology, Maria Middelares Hospital, Buitenring Sint-Denijs 30, 9000 Gent, Belgium.

\begin{abstract}
In this case report we present a family cluster of amoebiasis in a nonendemic region.

A 46-year-old women, diagnosed with Crohn's disease for which she received no maintenance therapy, was evaluated for the suspicion of a flare. At colonoscopy however, atypical findings for Crohn's disease were seen. Histopathologic examination revealed micro-organisms compatible with amoebiasis. Interestingly, 4 years before this event she started a new relationship with a 38 -year-old man who was diagnosed with liver-amoebiasis 3 months after the start of their relationship. On top of this, her 18-year-old daughter was diagnosed with amoebiasis 2 years after her diagnosis. The source of the infection remains unknown, but we speculate that the infection was transmitted feco-orally between the different members of this family. These cases illustrate that we should be aware of parasitological causes of colitis, especially in patients with atypical endoscopic images or when a close "relative" is diagnosed with amoebiasis. (Acta gastroenterol. belg., 2021, 84, 362-364).
\end{abstract}

Keywords : Amoebiasis, colitis, inflammatory bowel disease, colonoscopy, liver abscess.

\section{Introduction}

Entamoeba histolytica is a protozoan parasite with a global distribution and a high prevalence in developing countries where poor socioeconomic and sanitary conditions prevail (1). According to the definition of the World Health Organization (WHO), amoebiasis is an infection with Entamoeba histolytica, whether it causes symptoms or not (2). Most infections with Entamoeba histolytica appear to be asymptomatic (90\%), intestinal disease occurs in $10 \%$ and extraintestinal disease in less than $10 \%$ (3). The onset is subacute and patients can present with abdominal pain, bloody or watery and mucus containing diarrhea. A different presentation is chronic intestinal amoebiasis in which patients have periodic complaints for years, mimicking inflammatory bowel disease (IBD) $(4,5)$. Complications of intestinal disease are strictures, rectovaginal fistula, ameboma, perianal skin ulceration, bowel obstruction, toxic megacolon, perforation, peritonitis and death $(3,6)$. A liver abscess is the most frequent extraintestinal presentation, further dissemination to the brain, spleen, lungs, heart or genitourinary tract is possible $(3,6)$. It's important to distinguish amoebiasis from other diagnosis like IBD as soon as possible, to allow proper treatment and to avoid the development of complications.

In this paper we present 3 patients of the same family without a travel history to an endemic region or other

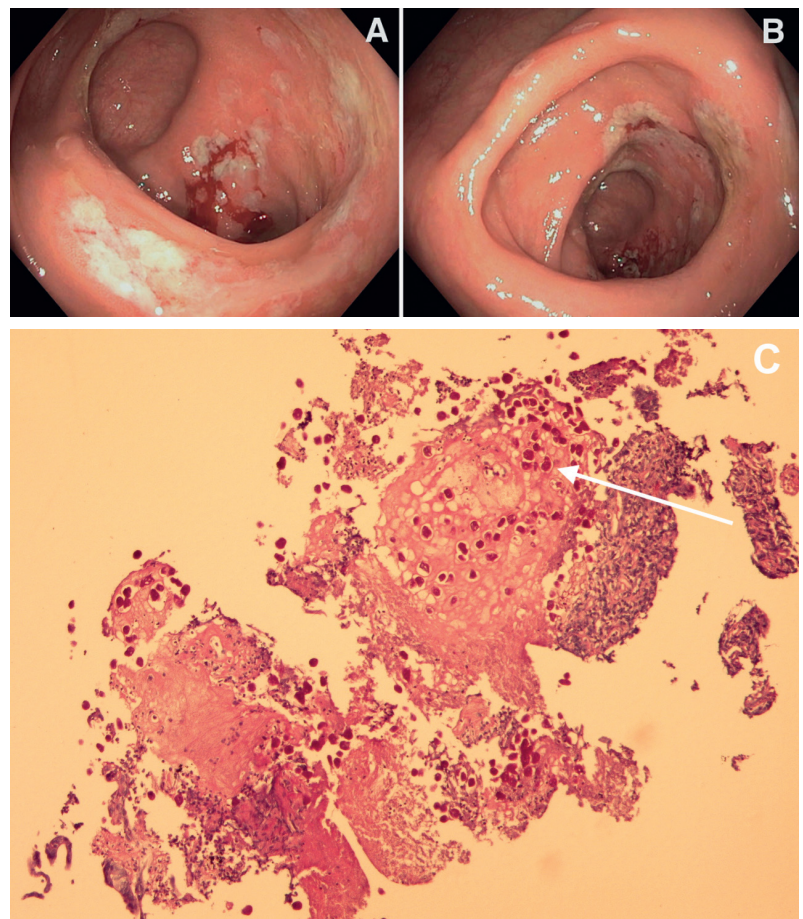

Figure 1. - Images during endoscopy, panel A : image of the caecum with skip lesions, multiple ulcers with a purulent film and shallow, irregular appearance ; panel B : image of the ascending colon with skip lesions ; panel C : Periodic AcidSchiff's (PAS) stain of the colonic biopsies: magenta coloring of the trophozoites (arrow).

risk factors. One of them presented with liver abscesses and the other 2 with a subacute presentation of intestinal amoebiasis.

\section{Cases}

\section{Patient 1}

A 46-year-old female presented with continuous cramping pain in her right lower abdomen, aggravating during the weeks before presentation. She had no travel history in the past years. Her bowel movements were

Correspondence to : Thomas De Somer, Buitenring Sint-Denijs 30, 9000 Gent, Belgium. Fax: 09/246.71.09

Email : thomasdesomer@gmail.com

Submission date : 04/06/2020

Acceptance date : 06/07/2020 
normal, she had no weight loss or fever. In her medical history, the patient was 11 years earlier diagnosed with Crohn's disease based on endoscopy with ulceration of the caecum and the ascending colon and suggestive histopathological examination, for which she received no maintenance therapy. Ultrasonography revealed a thickened wall of the cecum. On subsequent colonoscopy there were multiple shallow, irregular ulcerations, with friability of the mucosa in the cecum and the ascending colon (figure 1, panel A and B). Histopathologic examination of the ulcerations revealed eosinophilic granulocytes and micro-organisms, highly suggestive for amoebiasis. Periodic Acid-Schiff's (PAS) stain was performed and colored the trophozoites bright magenta (figure 1, panel C). Appropriate treatment with metronidazole followed by paromomycin was prescribed. Since she had no remaining symptoms after the treatment with metronidazole, she did not take the paromomycin as requested.

\section{Patient 2}

Four years before the diagnosis of amoebiasis and 3 months after the start of their relationship, the 38-yearold partner of the first patient was hospitalised because of fever of unknown origin. In his medical history he had psoriatic arthritis for which he was treated with methotrexate and cyclosporine. Ultrasound of the abdomen and additional computed tomography of the abdomen revealed 2 large liver lesions $(6.5 \times 7 \mathrm{~cm}$ in the left liver lobe and $8.5 \times 6.5 \mathrm{~cm}$ in the right liver lobe) (figure 2, panel A and B). Polymerase chain reaction (PCR) on punctate of one of the liver lesions was positive for Entamoeba histolytica, as well as serology. Diagnosis of amoebiasis was made and the patient was treated with metronidazole for 14 days followed by paromomycin with disappearance of the complaints and regression of the liver abscesses.

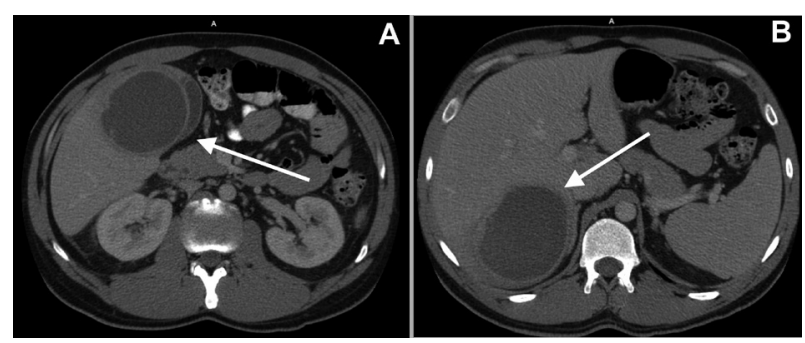

Figure 2. - Axial computed tomography images with contrast, panel A : image of the lesion in the left liver lobe (arrow); panel $\mathrm{B}$ : image of the lesion in the right liver lobe (arrow).

\section{Patient 3}

The 18-year-old daughter of the first patient presented about 2 years after the diagnosis of her mother with pain in the right lower abdomen and diarrhea. She reported no travelling in the past years. She had a blank medical history and took no medication. Ultrasonography of the abdomen revealed no abnormalities. During colonoscopy mild erythema with small erosions in the caecum and the ascending part of the colon were seen. On histopathological examination there were no arguments for amoebiasis, only active colitis. Microscopic investigation of a stool sample revealed cysts compatible with amoebiasis, serology for Entamoeba histolytica was positive, PCR on the biopsies in the colon was positive, as well as a PCR on the stool sample. She was treated with metronidazole followed by paromomycin. During the treatment the patient noticed an improvement of her symptoms and after the treatment all symptoms had disappeared. Re-evaluation of the mother revealed a positive PCR for Entamoeba histolytica on a stool sample. The partner of the mother was screened as well and had no arguments for recurrent amoebiasis.

\section{Discussion}

There are 2 forms of Entamoeba histolytica: the invasive trophozoites and the infectious cysts. The life cycle starts with the ingestion of Entamoeba histolytica cysts which survive the passage through the acidic stomach and the small intestine. Excystation occurs in the lumen of the colon where trophozoites are formed. In most infections, the trophozoites reproduce, aggregate and form new cysts in the intestinal mucin layer. Cysts are formed and excreted in stool for further transmission. However, rarely invasion is initiated when trophozoites adhere to the colonic epithelium. Invasive disease can occur months to years after ingestion $(3,6)$.

Feco-oral transmission by ingestion of contaminated food or water is the most frequent route of transmission (3). However, there are increasing data of sexual transmission as well (7). Cases are published with Entamoeba histolytica infections in men who have sex with man (MSM), especially when patients are infected with human immunodeficiency virus (HIV) $(7,8)$. A report from Canada could trace a cluster of 7 cases of Entamoeba histolytica in heterosexual and homosexual sexual partners with common oral-anal sex through 1 index case (9). Transmission in sexual intercourse is seen in oral-anal contact, anal sexual intercourse and fellatio after anal sexual intercourse (8).

Entamoeba species can be recognized with light microscopic examination of a stool sample, however the sensitivity is low (25-60\%) (3). Serology has a high sensitivity of $70 \%$ (early) to more than $90 \%$ (convalescent sample) and is of particular use in nonendemic regions because differentiation between a current or past infection is not possible (3). PCR can be performed on a stool sample, tissue biopsy and aspirate from an abscess, sensitivity and specificity are high: approximately $94 \%$ and $100 \%$ respectively $(3,10)$. Colonoscopy with histopathologic examination can exclude other diagnoses or can help to establish the diagnosis, the caecum and the ascending colon are most frequently involved (11). The 
endoscopic appearance of amoebiasis is variable, from normal over diffuse erythema, mucosal friability, erosions and multiple skip lesions with punctate ulcers, to the most extreme form of amoeboma (pseudotumoral aspect) $(11,12)$. Amoeboma occur rarely and are the result of the formation of granulation tissue. Sometimes it's difficult to differentiate an amoeboma from a carcinoma as illustrated with a recent case report (13). Histopathologic examination of biopsies obtained during colonoscopy reveal the presence of trophozoites or cysts and typical flask-shaped ulcers (3). Since the endoscopic findings may resemble those seen in IBD, differential diagnosis can be difficult $(4,5)$. Distinguishing amoebiasis from IBD is, however, important since the administration of corticosteroids in patients with amoebiasis may result in fulminant disease (12).

In our case the intestinal amoebiasis of the patient was at first misdiagnosed as a mild flare of her Crohn's disease. Only after the colonoscopy, the possibility of a parasitological cause was considered because of the atypical appearance during colonoscopy.

According to the WHO every symptomatic or asymptomatic patient with proven Entamoeba histolytica infection should be treated (2). More or less there are 2 sorts of amoebicides: luminal and tissue. The tissue amoebicides (nitroimidazoles) are effective in invasive amoebiasis, but luminal cysts and trophozoites can survive. Luminal amoebicides (for example paromomycin) are less effective in treating invasive amoebiasis, but treatment after a tissue amoebicide, improves the eradication of luminal cysts and trophozoites (3). Asymptomatic patients can be treated with a luminal amoebicide in monotherapy. A frequently used treatment regimen for invasive amoebiasis is metronidazole 500 to $750 \mathrm{mg} 3$ times a day during 7 to 10 days, followed by paromomycin $30 \mathrm{mg} / \mathrm{kg}$ daily divided in 3 doses during 7 days $(2,6)$.

In our family cluster we think that the first patient was an oligosymptomatic carrier who was able to infect her new partner through feco-oral or sexual transmission. The boyfriend was immunocompromised, which probably led to an invasive infection with large liver abscesses. The first patient was known to have Crohn's disease, but she never received treatment for it and had no symptoms, possibly she already had amoebiasis at the time, instead of IBD. When she was diagnosed, retrospectively, she never completed her treatment and the amoebiasis was therefore most likely never completely cured. Because of this, she was still infectious and was able to infect her daughter. Her partner and daughter were adequately treated.

\section{Conclusion}

Amoebiasis is a rare disease in the Western world and is mostly seen in patients who travelled to or immigrated from endemic regions. Diagnosis can be established with a whole range of diagnostic tests. Distinguishing amoebiasis from inflammatory bowel disease is often difficult, but possible with the combination of endoscopy and histopathologic examination of biopsies, combined with awareness of more uncommon etiologies of colitis. Amoebiasis is treatable with readily available luminal and tissue amoebicides. Our cases illustrate that parasitological causes of colitis should be kept in mind, even in patients who have not travelled to endemic regions and have no other risk factors.

\section{Conflicts of interest}

None declared.

\section{References}

1. STANLEY S.L., Jr. Amoebiasis. Lancet, 2003, 361 : 1025-1034.

2. WHO/PAHO/UNESCO REPORT. A consultation with experts on amoebiasis. Epidemiol. Bull., 1997, 18 : 13-14.

3. HAQUE R., HUSTON C.D., HUGHES M., HOUPT E., PETRI W.A., Jr. Amebiasis. N. Engl. J. Med., 2003, 348 : 1565-1573.

4. VERSTOCKT B., VERMEIRE S., VAN ASSCHE G., FERRANTE M. When IBD is not IBD. Scand. J. Gastroenterol., 2018, 530:1085-1088.

5. DEBOURDEAU A., BOIVINEAU L., ILTACHE S. The little beast that pretended to be a severe Crohn's disease. Gastroenterology, 2019, 157 : $1483-1484$.

6. ROSS A.G., OLDS G.R., CRIPPS A.W., FARRAR J.J., MCMANUS D.P. Enteropathogens and chronic illness in returning travelers. N. Engl. J. Med., 2013, 368 : 1817-1825.

7. WILLIAMSON D.A., CHEN M.Y. Emerging and reemerging sexually transmitted Infections. N. Engl. J. Med., 2020, 382 : 2023-2032.

8. HUNG C.C., CHANG S.Y., JI D.D. Entamoeba histolytica infection in men who have sex with men. Lancet Infect. Dis., 2012, 12 : 729-736.

9. SALIT I.E., KHAIRNAR K., GOUGH K., PILLAI D.R. A possible cluster of sexually transmitted Entamoeba histolytica: genetic analysis of a highly virulent strain. Clin. Infect. Dis., 2009, 49 : 346-353.

10. CHOUDHURI G., RANGAN M. Amebic infection in humans. Indian $J$. Gastroenterol., 2012, 31 : 153-162.

11. LEE K.C., LU C.C., HU W.H., LIN S.E., CHEN H.H. Colonoscopic diagnosis of amebiasis: a case series and systematic review. Int. J. Colorectal Dis., 2015, $30: 31-41$.

12. LI N., WANG H.H., ZHAO X.J., SHENG J.Q. Amebic colitis : colonoscopic appearance. Endoscopy, 2015, 47 : E145-146.

13. BRONSWIJK M., VAN GOOL S. A case of amoebic colitis with amoeboma and simultaneous liver abscesses. A diagnosis by colonoscopy. Acta Gastroenterol. Belg., 2019, 82 : 539-41. 